\title{
Semidefinite Relaxation for Detection of 16-QAM Signaling in MIMO Channels
}

\author{
Ami Wiesel, Student Member, IEEE, Yonina C. Eldar, Member, IEEE, and Shlomo Shamai (Shitz), Fellow, IEEE
}

\begin{abstract}
We develop a computationally efficient approximation of the maximum likelihood (ML) detector for 16 quadrature amplitude modulation (16-QAM) in multiple-input multiple-output (MIMO) systems. The detector is based on a convex relaxation of the $M L$ problem. The resulting optimization is a semidefinite program that can be solved in polynomial time with respect to the number of inputs in the system. Simulation results in a random MIMO system show that the proposed algorithm outperforms the conventional decorrelator detector by about 2.5 $\mathrm{dB}$ at high signal-to-noise ratios.
\end{abstract}

Index Terms-Maximum likelihood detection, MIMO systems, semidefinite relaxation.

\section{INTRODUCTION}

$\mathbf{M}$ ULTIPLE-INPUT multiple-output (MIMO) systems arise in many modern communication channels, such as multiple user communication and multiple antennas channels. It is well known that the use of multiple transmit and receive antennas promises substantial performance gains when compared to traditional single antenna systems. In order to exploit these gains, the system must be able to efficiently detect the transmitted symbols at the receiver. Hence, detection in MIMO systems is one of the fundamental problems in state-of-the-art communication systems.

The optimal algorithm in the sense of minimum joint probability of error for detecting all the symbols simultaneously is the maximum likelihood (ML) detector [1]. It can be implemented using a brute-force search over all of the possible transmitted vectors or using more efficient search algorithms, e.g., the sphere decoder [2]. However, it has been shown that even when the sphere decoder is used, the expected computational complexity is exponential and impractical for many applications [3]. Consequently, there has been much interest in implementing suboptimal detection algorithms. The most common suboptimal detectors are the linear receivers, i.e., the matched filter (MF), the decorrelator or zero forcing (ZF), and the minimum mean-squared error (MMSE) detectors. More advanced detectors are based on decision feedback equalization (DFE). There are dozens of other suboptimal detection schemes ranging from lattice-based algorithms, alternating variable methods, to

Manuscript received December 30, 2004; revised February 28, 2005. This work was supported by the European Union 6th framework programme, via the NEWCOM network of excellence, and by the ISRAEL SCIENCE FOUNDATION by the Israel Academy of Sciences and Humanities. The associate editor coordinating the review of this manuscript and approving it for publication was Dr. Dominic K. C. Ho.

The authors are with the Department of Electrical Engineering, Technion, Israel Institute of Technology, Haifa 32000, Israel (e-mail: amiw@tx.technion.ac.il; yonina@ee.technion.ac.il; sshlomo@ee.technion.ac.il).

Digital Object Identifier 10.1109/LSP.2005.853044 expectation maximization and many more (see [1], [4], and references within).

One of the most promising suboptimal detection strategies is the semidefinite relaxation (SDR) detector, which recently gained considerable attention [4]-[11]. The main reason for the high computational complexity of the ML detector is due to the fact that it is a non convex optimization problem. SDR is an attempt to approximate it using a convex program that can be efficiently solved in polynomial time. There are two approaches for deriving the SDR. The first approach is to formulate the ML problem in a higher dimension and then relax the nonconvex constraints. Alternatively, the SDR detector can be derived as the Lagrange bidual of the ML optimization problem, i.e., the dual program of the dual of the ML problem (e.g., [12, Exercise 5.39]). The resulting SDR in both of these approaches is a semidefinite program (SDP) for which there are efficient solutions that can be obtained in polynomial time [13].

Initially, the SDR was proposed for detection of binary/quadratic phase shift keying (BPSK/QPSK) constellations [4]-[6]. In this simple signaling scheme, simulation results show that the SDR provides near-ML performance [5]. Conditions for the tightness of the SDR in the case of BPSK were derived in [7]. These suggest that at high signal-to-noise ratios (SNRs), there is a high probability that SDR will yield the true ML decision. Other results that motivate the use of the SDR show that many of the other conventional detectors, such as the MMSE, are relaxations of the SDR and are, therefore, inferior (at least before the discretization) [5]. There are many works on practical low-complexity implementations of the SDR algorithm that are suitable for MIMO channels with a large number of multiple inputs [6], [9], [10]. Another important feature of the SDR is that its solution can be easily modified to provide soft decisions as required in state-of-the-art communication systems [8].

The success of the SDR in demodulating BPSK signaling motivated its generalization to higher constellations. In [10] and [11], the SDR was generalized to the detection of M-phase shift keying (M-PSK) signaling. In our letter, we propose to extend these works to the detection of other constellations used in digital communications. The key observation is that any finite alphabet constraint can be replaced by a polynomial constraint, e.g., if $x \in\{a, b, c\}$, then $(x-a)(x-b)(x-c)=0$. Next, by introducing slack variables, the high-order polynomial constraint can be replaced by multiple quadratic constraints. These constraints can be convexified by either reformulating them in a higher dimension and relaxing or by deriving the Lagrange bidual. For simplicity, we restrict ourselves to the well-used 16 quadrature amplitude modulation (16-QAM) constellation set. 
The same general approach can be followed for other constellations.

The letter is organized as follows. In Section II, we introduce the 16-QAM detection problem in MIMO channels and review the well known ML detector. In Section III, we derive the 16-QAM SDR detector based on rank relaxation. An alternative derivation based on the Lagrange bidual is provided in Section IV. The advantage of the SDR detector is demonstrated in Section V using computer simulations. Finally, in Section VI, we provide concluding remarks.

The following notation is used. Boldface uppercase letters denote matrices, boldface lowercase letters denote column vectors, and standard lowercase letters denote scalars. The superscript $(\cdot)^{T}$ denotes the transpose. $\mathbf{x}_{i}$ denotes the $i$ th element of the vector $\mathbf{x}$. By Diag $\left\{x_{i}\right\}$, we denote a diagonal matrix with $x_{i}$ being the (ith, $i$ th) element, by diag $\{\mathbf{X}\}$, we denote the vector comprised of the diagonal elements of the matrix $\mathbf{X}$, by $\mathbf{I}$, we denote the identity matrix, and by $\mathbf{1}$, we denote the vector of ones. $\mathcal{R} e\{\cdot\}$ and $\mathcal{I} m\{\cdot\}$ denote the real part and the imaginary part, respectively. $\operatorname{Tr}\{\cdot\}$ denotes the trace operator, and $\|\cdot\|$ denotes the standard Euclidean norm. Finally, $\mathbf{X} \succeq 0$ means that the matrix $\mathbf{X}$ is a Hermitian positive semidefinite matrix.

\section{16-QAM ML DETECTION}

Consider the standard MIMO channel

$$
\overline{\mathbf{y}}=\overline{\mathbf{H}} \overline{\mathbf{s}}+\overline{\mathbf{w}}
$$

where $\overline{\mathbf{y}}$ is the received signal of length $N, \overline{\mathbf{H}}$ is an $N \times K$ channel matrix, $\overline{\mathbf{s}}$ is the length $K$ vector of transmitted symbols, and $\overline{\mathbf{w}}$ is a length $N$ complex normal zero-mean noise vector with covariance $\sigma^{2} \mathbf{I}$. The symbols of $\overline{\mathbf{s}}$ belong to some known complex constellation. In this letter, we consider the 16-QAM constellation, i.e., the real part and the imaginary part of $\overline{\mathbf{s}}_{i}$ for $i=1, \ldots, K$ belong to the set $\{ \pm 1, \pm 3\}$.

In order to avoid the need to handle complex-valued variables, it is customary to use the following decoupled model:

$$
\mathbf{y}=\mathbf{H s}+\mathbf{w}
$$

where

$$
\begin{aligned}
& \mathbf{y}=\left[\begin{array}{c}
\mathcal{R} e\{\overline{\mathbf{y}}\} \\
\mathcal{I} m\{\overline{\mathbf{y}}\}
\end{array}\right] ; \mathbf{s}=\left[\begin{array}{c}
\mathcal{R} e\{\overline{\mathbf{s}}\} \\
\mathcal{I} m\{\overline{\mathbf{s}}\}
\end{array}\right] ; \mathbf{w}=\left[\begin{array}{c}
\mathcal{R} e\{\overline{\mathbf{w}}\} \\
\mathcal{I} m\{\overline{\mathbf{w}}\}
\end{array}\right] ; \\
& \mathbf{H}=\left[\begin{array}{cc}
\mathcal{R} e\{\overline{\mathbf{H}}\} & -\mathcal{I} m\{\overline{\mathbf{H}}\} \\
\mathcal{I} m\{\overline{\mathbf{H}}\} & \mathcal{R} e\{\overline{\mathbf{H}}\}
\end{array}\right] .
\end{aligned}
$$

Using these definitions, the ML detector of the transmitted symbols is

$$
\mathrm{ML}:\left\{\begin{array}{l}
\min _{\mathbf{s}}\|\mathbf{y}-\mathbf{H s}\|^{2} \\
\text { s.t. } \mathbf{s}_{i} \in\{ \pm 1, \pm 3\}, \quad i=1, \ldots, 2 K .
\end{array}\right.
$$

The program ML is a combinatorial problem and can be solved in a brute-force fashion by searching over all of the $4^{2 K}=16^{K}$ possibilities. Clearly, as $K$ increases, this option becomes impractical. In the next section, we propose an approximate solution to the problem via semidefinite relaxation.

\section{SDR VIA RANK RELAXATION}

The key observation that leads to the SDR is that the constraint $\mathbf{s}_{i} \in\{ \pm 1, \pm 3\}$ for $i=1, \ldots, 2 K$ can be expressed as

$$
\left(s_{i}+1\right)\left(s_{i}-1\right)\left(s_{i}+3\right)\left(s_{i}-3\right)=0, \quad i=1, \ldots, 2 K .
$$

Introducing the slack variables $t_{i}=s_{i}^{2}$ for $i=1, \ldots, 2 K$, we can use (5) to rewrite the problem ML as

$$
\begin{cases}\min _{\mathbf{s}, \mathbf{t}} & \|\mathbf{y}-\mathbf{H s}\|^{2} \\ \text { s.t. } & s_{i}^{2}-t_{i}=0, \quad i=1, \ldots, 2 K \\ & t_{i}^{2}-10 t_{i}+9=0, \quad i=1, \ldots, 2 K .\end{cases}
$$

The next step in deriving the SDR is formulating the optimization problem in a higher dimension. We replace the vectors $\mathbf{s}$ and $\mathbf{t}$ with a rank-one semidefinite matrix $\mathbf{W}=\mathbf{w w}^{T}$, where $\mathbf{w}^{T}=\left[\begin{array}{lll}\mathbf{s}^{T} & \mathbf{t}^{T} & 1\end{array}\right]$. Using this change of variables, we can easily identify $\mathbf{W}_{1,1}=\mathbf{s s}^{T}, \quad \mathbf{W}_{2,2}=\mathbf{t t}^{T}, \quad \mathbf{W}_{1,3}=\mathbf{s}$, and $\quad \mathbf{W}_{2,3}=\mathbf{t}$, where $\mathbf{W}_{i, j}$, for $i, j=1,2,3$, are the $(i, j)$ th sub-blocks of $\mathbf{W}$ of appropriate sizes. Therefore, problem (6) is equivalent to

$$
\left\{\begin{aligned}
\min _{\mathbf{W}} & \operatorname{Tr}\left\{\mathbf{W}\left[\begin{array}{ccc}
\mathbf{H}^{T} \mathbf{H} & \mathbf{0} & -\mathbf{H}^{T} \mathbf{y} \\
\mathbf{0} & \mathbf{0} & \mathbf{0} \\
-\mathbf{y}^{T} \mathbf{H} & \mathbf{0} & \mathbf{y}^{T} \mathbf{y}
\end{array}\right]\right\} \\
\text { s.t. } & \operatorname{diag}\left\{\mathbf{W}_{1,1}\right\}-\mathbf{W}_{2,3}=\mathbf{0} \\
& \operatorname{diag}\left\{\mathbf{W}_{2,2}\right\}-10 \mathbf{W}_{2,3}+91=\mathbf{0} \\
& \mathbf{W} \succeq 0 \\
& \mathbf{W}_{3,3}=1 \\
& \operatorname{rank}(\mathbf{W})=1 .
\end{aligned}\right.
$$

The program (7) is not convex because of the rank-one constraint. Dropping this constraint results in the SDR

$$
\text { SDR : }\left\{\begin{aligned}
\min _{\mathbf{W}} \operatorname{Tr}\left\{\mathbf{W}\left[\begin{array}{ccc}
\mathbf{H}^{T} \mathbf{H} & \mathbf{0} & -\mathbf{- H}^{T} \mathbf{y} \\
\mathbf{0} & \mathbf{0} & \mathbf{0} \\
-\mathbf{y}^{T} \mathbf{H} & \mathbf{0} & \mathbf{y}^{T} \mathbf{y}
\end{array}\right]\right\} \\
\text { s.t. } \operatorname{diag}\left\{\mathbf{W}_{1,1}\right\}-\mathbf{W}_{2,3}=\mathbf{0} \\
\quad \operatorname{diag}\left\{\mathbf{W}_{2,2}\right\}-10 \mathbf{W}_{2,3}+91=\mathbf{0} \\
\mathbf{W} \succeq 0 \\
\mathbf{W}_{3,3}=1 .
\end{aligned}\right.
$$

Note that the SDR has a linear objective subject to affine equalities and a linear matrix inequality. Such problems are known as SDP and can be efficiently solved in polynomial time [13], [14].

If the optimal argument $\mathbf{W}$ of SDR has rank one, then the relaxation is tight, and the ML solution of $\mathbf{s}$ is the first $2 K$ elements of the last column of $\mathbf{W}$. Otherwise, SDR is only an approximation of ML, and there is no strict relation between $\mathbf{W}$ and s. Instead, there are a few standard techniques for approximating $\mathbf{s}$ based on $\mathbf{W}[10]$ :

\section{- Simple quantization:}

$$
\widehat{s}_{i}=\text { quantize }\left(\mathbf{W}_{i, 4 K+1}\right), \quad i=1, \ldots, 2 K
$$

where quantize $(\alpha)$ rounds $\alpha$ to the nearest element in the set $\{ \pm 1, \pm 3\}$. 
- $\quad$ Eigenvalue decomposition: Let $\mathbf{u}$ denote the eigenvector of

$$
\widetilde{\mathbf{W}}=\left[\begin{array}{cc}
\mathbf{W}_{1,1} & \mathbf{W}_{1,3} \\
\mathbf{W}_{3,1} & 1
\end{array}\right]
$$

associated with its maximal eigenvalue. Then

$$
\widehat{s}_{i}=\text { quantize }\left(\frac{\mathbf{u}_{i}}{\mathbf{u}_{2 K+1}}\right), \quad i=1, \ldots, 2 K .
$$

- Randomization: Let $\widetilde{\mathbf{W}}=\mathbf{V}^{T} \mathbf{V}$ be the Cholesky factorization of $\widetilde{\mathbf{W}}$ from (10), and denote the columns of $\mathbf{V}$ by $\mathbf{v}_{i}$. Then

$\widehat{s}_{i}=$ quantize $\left(\frac{\mathbf{v}_{i}^{T} \mathbf{r}}{\mathbf{v}_{2 K+1}^{T} \mathbf{r}}\right), \quad i=1, \ldots, 2 K$

where $\mathbf{r}$ is a random vector uniformly distributed on a $(2 K+1)$-dimensional unit sphere. In order to improve the approximation quality, the randomization is repeated a number of times, and the solution yielding the best objective value is chosen.

It is important to note that the above discretization methods are purely ad hoc. One may definitely derive other and maybe better methods. For example, by taking into account the constraints (8b) and (8c) and using some least-squares procedure, one can improve the quantization step. However, such methods are outside the scope of this letter.

\section{SDR AS THE LAGRANGE BIDUAL PROGRAM}

In the previous section, we derived the SDR by relaxing the nonconvex rank-one constraint. There is no rigorous mathematical justification or any sort of optimality associated with this relaxation. In this section, we show that the resulting SDR is, in fact, the Lagrange bidual of (6). For any optimization problem (not necessarily convex), there is a convex Lagrange dual program. The optimal value of the dual program is a bound on the optimal value of the original program. If the original problem was convex, then the bidual is usually the original problem itself (or a very similar problem with some change of variables). In nonconvex programs, the bidual cannot be exactly the original problem, since it is always a convex program. Therefore, the bidual is considered as a standard technique to convexify nonconvex problems. We now prove that the bidual of (6) results in the same SDR of (8).

The Lagrangian associated with program (6) is

$$
\begin{aligned}
L=\mathbf{s}^{T} \mathbf{H}^{T} \mathbf{H s}-2 \mathbf{y}^{T} \mathbf{H s}+\mathbf{y}^{T} \mathbf{y} \\
\quad+\sum_{j} d_{j}\left(t_{j}^{2}-10 t_{j}+9\right)+\sum_{j} r_{j}\left(s_{j}^{2}-t_{j}\right) .
\end{aligned}
$$

By introducing

$$
\begin{aligned}
\mathbf{Q}(\mathbf{d}, \mathbf{r}) & =\left[\begin{array}{cc}
\mathbf{H}^{T} \mathbf{H}+\operatorname{Diag}\{\mathbf{r}\} & \mathbf{0} \\
\mathbf{0} & \operatorname{Diag}\{\mathbf{d}\}
\end{array}\right] \\
\mathbf{f}^{T}(\mathbf{d}, \mathbf{r}) & =\left[\begin{array}{ll}
\mathbf{y}^{T} \mathbf{H} & 5 \mathbf{d}^{T}+\frac{1}{2} \mathbf{r}^{T}
\end{array}\right] \\
c(\mathbf{d}, \mathbf{r}) & =9 \mathbf{1}^{T} \mathbf{d}+\mathbf{y}^{T} \mathbf{y}
\end{aligned}
$$

the Lagrangian is a quadratic form in $\mathbf{x}=\left[\begin{array}{ll}\mathbf{s}^{T} & \mathbf{t}^{T}\end{array}\right]^{T}$

$$
L=\mathbf{x}^{T} \mathbf{Q}(\mathbf{d}, \mathbf{r}) \mathbf{x}-2 \mathbf{f}^{T}(\mathbf{d}, \mathbf{r}) \mathbf{x}+c(\mathbf{d}, \mathbf{r}) .
$$

The dual program of (6) is defined as

$$
\mathrm{D}:\left\{\max _{\mathbf{d}, \mathbf{r}} \min _{\mathbf{x}}\left\{\mathbf{x}^{T} \mathbf{Q}(\mathbf{d}, \mathbf{r}) \mathbf{x}-2 \mathbf{f}^{T}(\mathbf{d}, \mathbf{r}) \mathbf{x}+c(\mathbf{d}, \mathbf{r})\right\} .\right.
$$

Adding a slack variable $t$ yields

$$
\mathrm{D}:\left\{\begin{array}{l}
\max _{\mathbf{d}, \mathbf{r}, t} t \\
\text { s.t. } \mathbf{x}^{T} \mathbf{Q}(\mathbf{d}, \mathbf{r}) \mathbf{x}-2 \mathbf{f}^{T}(\mathbf{d}, \mathbf{r}) \mathbf{x} \\
\quad+c(\mathbf{d}, \mathbf{r}) \geq t, \quad \forall \mathbf{x} .
\end{array}\right.
$$

We now rely on the following well-known lemma.

Lemma 1: [13, p. 163] Let $\mathbf{A}$ be a symmetric matrix. The condition $\mathbf{x}^{T} \mathbf{A} \mathbf{x}+2 \mathbf{b}^{T} \mathbf{x}+c \geq 0$ holds for all $\mathbf{x}$ if and only if the matrix $\left[\begin{array}{cc}\mathbf{A} & \mathbf{b} \\ \mathbf{b}^{T} & c\end{array}\right] \succeq 0$ is semidefinite positive.

Due to the lemma, the dual program can be expressed as

$$
\mathrm{D}:\left\{\begin{array}{l}
\max _{\mathbf{d}, \mathbf{r}, t} t \\
\text { s.t. }\left[\begin{array}{cc}
\mathbf{Q}(\mathbf{d}, \mathbf{r}) & -\mathbf{f}(\mathbf{d}, \mathbf{r}) \\
-\mathbf{f}^{T}(\mathbf{d}, \mathbf{r}) & c(\mathbf{d}, \mathbf{r})-t
\end{array}\right] \succeq 0 .
\end{array}\right.
$$

We now derive the dual of the dual program. The Lagrangian associated with program $\mathrm{D}$ is

$$
\begin{aligned}
L_{D}= & -t-\operatorname{Tr}\left\{\mathbf{W}\left[\begin{array}{cc}
\mathbf{Q}(\mathbf{d}, \mathbf{r}) & \mathbf{f}(\mathbf{d}, \mathbf{r}) \\
-\mathbf{f}^{T}(\mathbf{d}, \mathbf{r}) & c(\mathbf{d}, \mathbf{r})-t
\end{array}\right]\right\} \\
= & t\left(-1+\mathbf{W}_{3,3}\right)-\mathbf{r}^{T}\left[\operatorname{diag}\left\{\mathbf{W}_{1,1}\right\}-\mathbf{W}_{2,3}\right] \\
& -\mathbf{d}^{T}\left[\operatorname{diag}\left\{\mathbf{W}_{2,2}\right\}-10 \mathbf{W}_{2,3}+9 \mathbf{W}_{3,3} \mathbf{1}\right] \\
& -\operatorname{Tr}\left\{\mathbf{W}_{1,1} \mathbf{H}^{T} \mathbf{H}\right\}+2 \mathbf{y}^{T} \mathbf{H} \mathbf{W}_{1,3}-\mathbf{W}_{3,3} \mathbf{y}^{T} \mathbf{y} .
\end{aligned}
$$

Linear terms are bounded from below only if they are identically zero. Therefore, the dual of (18) [which is also the bidual of (6)] is

$$
\text { DD : }\left\{\begin{array}{l}
\min _{\mathbf{W}} \operatorname{Tr}\left\{\mathbf{W}\left[\begin{array}{ccc}
\mathbf{H}^{T} \mathbf{H} & \mathbf{0} & -\mathbf{- H}^{T} \mathbf{y} \\
\mathbf{0} & \mathbf{0} & \mathbf{0} \\
-\mathbf{y}^{T} \mathbf{H} & \mathbf{0} & \mathbf{y}^{T} \mathbf{y}
\end{array}\right]\right\} \\
\text { s.t. } \operatorname{diag}\left\{\mathbf{W}_{1,1}\right\}-\mathbf{W}_{2,3}=\mathbf{0} \\
\operatorname{diag}\left\{\mathbf{W}_{2,2}\right\}-10 \mathbf{W}_{2,3}+9 \mathbf{W}_{3,3} \mathbf{1}=\mathbf{0} \\
\mathbf{W} \succeq 0 \\
\mathbf{W}_{3,3}=1
\end{array}\right.
$$

which is identical to the SDR of (8).

\section{Simulation Results}

In this section, we demonstrate the power of the SDR detector in an MIMO system using computer simulations. In our first simulation, we considered an MIMO system with $K=6$ inputs and $N=12$ outputs using 16-QAM signaling. The entries of 


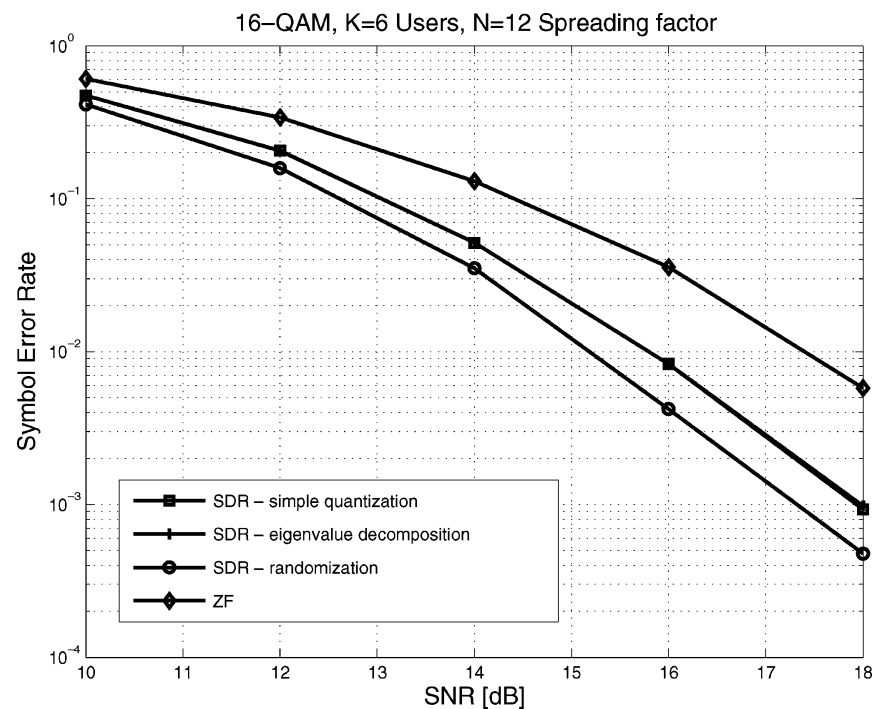

Fig. 1. Message error rates of a random MIMO system with $K=6$ and $N=$ 12 using 16-QAM

the MIMO channel were chosen as independent and identically distributed, zero-mean, complex normal random variables. For each SNR, we used up to 50000 Monte Carlo simulations in order to estimate the average probability of error in detecting the message vector. The three versions of the SDR detector presented in Section III were considered, where the randomization technique was implemented using 50 independent randomizations. The semidefinite program in the SDR was solved using the SeDuMi package [14]. For comparison, we also simulated the conventional linear ZF detector. The results are provided in Fig. 1. It is easy to see the advantage of the SDR detectors over the ZF detector. Among the SDR detectors, the randomization strategy is superior to the other two techniques, which perform roughly the same. Using this method, SDR gains up to $2.5 \mathrm{~dB}$ in high SNR when compared to the ZF strategy.

In the first simulation, we did not compare the performance of the SDR with that of the ML detector. This was due to the large number of possible vectors in the ML search $\left(16^{6}=16777216\right.$ vectors $)$. Therefore, in our second simulation, we consider a smaller system where a full ML search is possible. In particular, we used $K=4$ and $N=8$, i.e., $16^{4}=65536$ vectors in the ML search. The rest of the parameters are as in the first simulation. The results are provided in Fig. 2. As before, the SDR detector outperforms the ZF detector. Unfortunately, it can be observed that unlike the reported performance of the SDR in BPSK systems, in 16-QAM, the ML detector is still considerably better than the SDR detector.

\section{CONCLUSION}

In this letter, we derived the SDR of the ML detector for 16-QAM signaling over MIMO channels. Previously, the SDR was constrained to the detection of PSK signaling. Our approach shows that other digital constellations, such as QAM, can also be addressed using SDR by formulating the constraints set as multiple quadratic constraints and relaxing. Therefore, we find

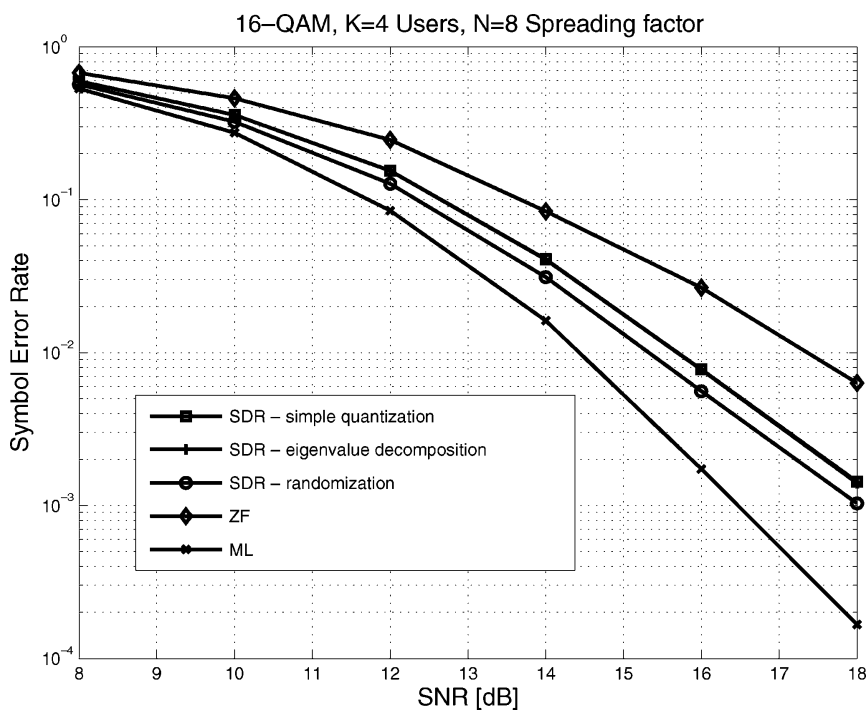

Fig. 2. Message error rates of a random MIMO system with $K=4$ and $N=$ 8 using 16-QAM in comparison to the ML detector.

the computationally efficient SDR detector as a competitive detector in comparison to other suboptimal methods.

\section{REFERENCES}

[1] S. Verdu, Multiuser Detection. Cambridge, U.K.: Cambridge Univ. Press, 1998.

[2] E. Agrell, T. Eriksson, A. Vardy, and K. Zeger, "Closest point search in lattices," IEEE Trans. Inf. Theory, vol. 48, no. 8, pp. 2201-2214, Aug. 2002.

[3] J. Jalden and B. Ottersten, "An exponential lower bound on the expected complexity of sphere decoding," in Proc. IEEE Int. Conf. Acoust., Speech, Signal Process., vol. 4, May 2004, pp. 393-396.

[4] P. H. Tan and L. K. Rasmussen, "The application of semidefinite programming for detection in CDMA," IEEE J. Sel. Areas Commun., vol. 19, no. 8, pp. 1442-1449, Aug. 2001.

[5] W. K. Ma, T. N. Davidson, K. M. Wong, Z. Q. Luo, and P. C. Ching, "Quasimaximum-likelihood multiuser detection using semi-definite relaxation with application to synchronous CDMA," IEEE Trans. Signal Process., vol. 50, no. 4, pp. 912-922, Apr. 2002.

[6] X. M. Wang, W. S. Lu, and A. Antoniou, "A near optimal multiuser detector for DS-CDMA systems using semidefinite programming relaxation," IEEE Trans. Signal Process., vol. 51, no. 9, pp. 2446-2450, Sep. 2003.

[7] J. Jalden, C. Martin, and B. Ottersten, "Semidefinite programming for detection in linear systems-Optimality conditions and space-time decoding," in Proc. IEEE Int. Conf. Acoust., Speech, Signal Process., vol. 4, Apr. 2003, pp. 9-12.

[8] B. Steingrimsson, Z. Q. Luo, and K. M. Wong, "Soft quasimaximum-likelihood detection for multiple-antenna wireless channels," IEEE Trans. Signal Process., vol. 51, no. 11, pp. 2710-2719, Nov. 2003.

[9] M. Abdi, H. E. Nahas, A. Jard, and E. Moulines, "Semidefinite positive relaxation of the maximum likelihood criterion applied to multiuser detection in CDMA context," IEEE Signal Process. Lett., vol. 9, no. 6, pp. 165-167, Jun. 2002.

[10] W. K. Ma, P. C. Ching, and Z. Ding, "Semidefinite relaxation based multiuser detection for M-ary PSK multiuser systems," IEEE Trans. Signal Process., vol. 52, no. 10, pp. 2862-2872, Oct. 2004.

[11] J. Dahl, B. H. Fleury, and L. Vandenberghe, "Approximate maximum-likelihood estimation using semidefinite programming," in Proc. IEEE Int. Conf. Acoust., Speech, Signal Process., vol. 6, Apr. 2003, pp. 721-724.

[12] S. Boyd and L. Vandenberghe, Introduction to Convex Optimization with Engineering Applications. Stanford, CA: Stanford Univ. Press, 2003.

[13] A. Ben Tal and A. Nemirovski, "Lectures on modern convex optimization," MPS-SIAM Series on Optimization, 2001.

[14] J. F. Sturm, "Using SEDUMI 1.02, A Matlab toolbox for optimizations over symmetric cones," Optim. Meth. Soft., vol. 11-12, 1999. 